\title{
ESPECIES LEÑOSAS DE LA SIERRA DE LOS CUCHUMATANES Y DE LA CADENA VOLCANICA, GUATEMALA
}

\author{
Gerald A. Islebe \\ Antolne M. Cleef \\ Laboratorio Hugo de Vries \\ Universidad de Amsterdam \\ Kruislaan 318 \\ 1098 SM Amsterdam, Holanda \\ Y \\ Alejandro Velazquez \\ Laboratorio de Biogeografía \\ Facultad de Ciencias, UNAM \\ 04510 México, D.F.
}

\section{RESUMEN}

El presente listado registra 112 especies de plantas leñosas encontradas entre 3000 y 4000 $\mathrm{m}$ de altitud en la Sierra de los Cuchumatanes y en la Cadena Volcánica de Guatemala, agrupadas en 39 familias y 63 géneros diferentes. Las familias mejor representadas son Asteraceae y Ericaceae.

\section{ABSTRACT}

The present checklist reports 112 woody plant species found in the Sierra de los Cuchumatanes and the Volcanic Chain of Guatemala (elev. 3000-4000 m). These 112 species belong to 39 families and 63 genera. The best represented families are Asteraceae and Ericaceae.

\section{INTRODUCCION}

Standley y Steyermak (1945) estimaron que Guatemala alberga alrededor de 8,000 especies de plantas vasculares. Esto sugiere que el país aparezca como uno de los de mayor diversidad florística en la región Meso-Americana. Wagner (1866) inicia la descripción de este universo vegetal guatemalteco, seguido por Sapper (1894, 1897, 1902), Rodríguez (1921) y la serie de publicaciones de Smith (1889-1907). Standley et al. (1946-1977) elaboraron contribuciones extensas en la "Flora of Guatemala". Simultáneamente Bartram (1949) documentó la diversidad de briófitas en su contribución denominada "Mosses of Guatemala". Más recientemente, Gentry (1978) argumenta que existen registros prácticamente completos de la flora guatemalteca y propone regionalizar el país con base en la composición florística de cada región. En contraste con el buen conocimiento de Guatemala, 
aún son escasos los estudios que definan en detalle la flora de la Sierra de los Cuchumatanes y que además permitan realizar comparaciones con otras áreas de MesoAmérica.

En este listado se presenta un inventario de las especies leñosas de la vegetación de la Sierra de los Cuchumatanes y de la Cadena Volcánica, que pueden encontrarse entre 3000 y 4000 m s.n.m.

\section{AREA DE ESTUDIO}

La Sierra de los Cuchumatanes, situada al noroeste de Guatemala, es la cadena montañosa donde se encuentran algunas de las cimas de mayor elevación de la parte septentrional de Centroamérica (Fig. 1). La cumbre más alta, localizada en el municipio de Todos Santos Cuchumatán (Xemal), alcanza los 3829 m s.n.m. La zonación altitudinal de la vegetación se presenta de la siguiente manera: de los 2900 a los 3400 m predominan

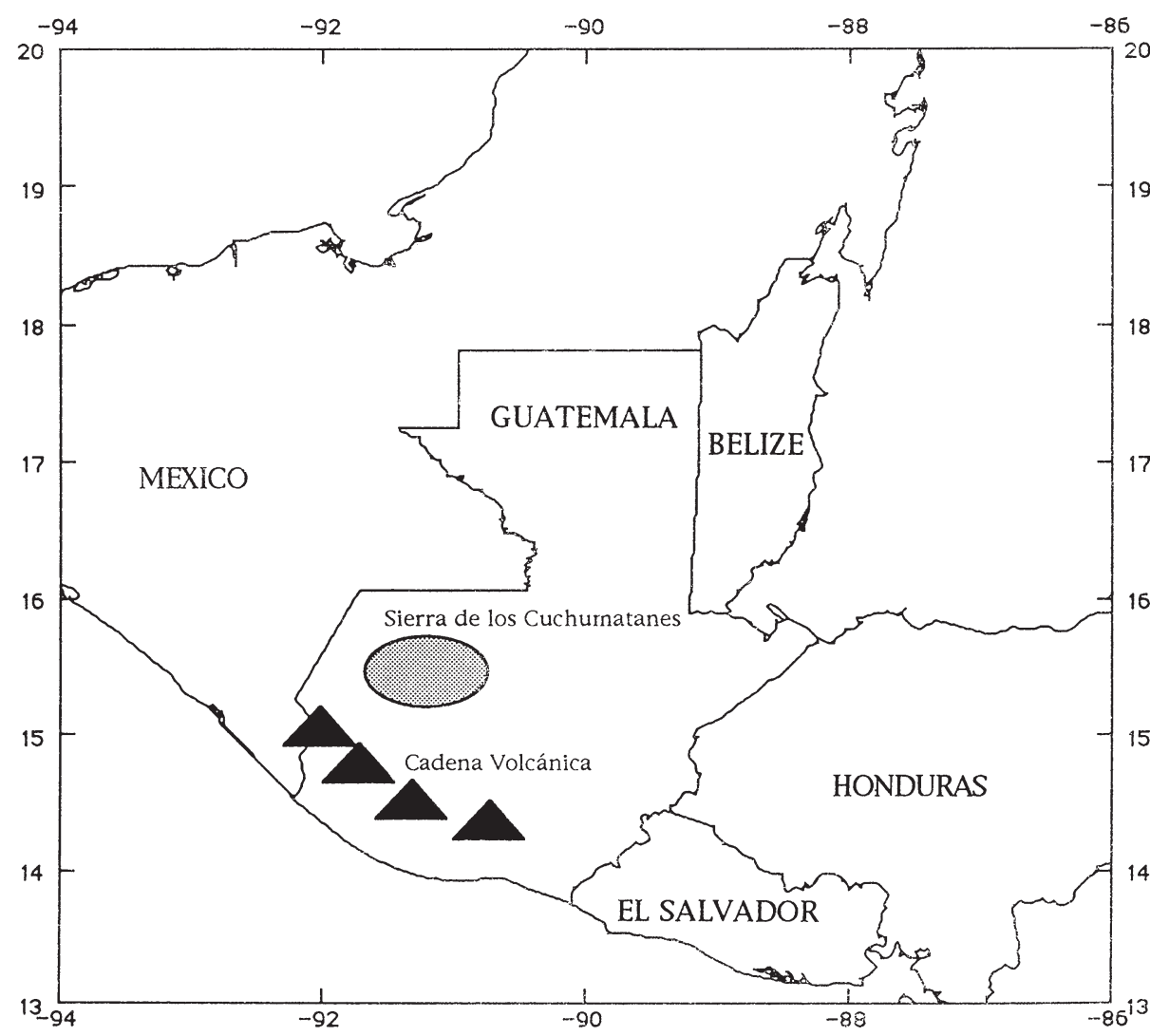

Fig. 1. Localización de la Sierra de los Cuchumatanes y de la Cadena Volcánica, Guatemala. 
Islebe et al.: Especies Leñosas de la Sierra de los Cuchumatanes y de la Cadena Volcánica

los bosques de Abies guatemalensis, mientras que de los 3000 a los $3800 \mathrm{~m}$ prevalece un bosque mixto de Juniperus standleyi-Pinus hartwegii. La distribución de estos cinturones de vegetación puede variar en función de factores edáficos y climatológicos, por ejemplo, la comunidad de $A$. guatemalensis es favorecida por condiciones de mayor humedad mientras que la de $J$. standleyi-P. hartwegii es propia de lugares más secos.

Los picos más altos de Centroamérica, representados por los volcanes Tajumulco (4220 m), Tacaná (4093 m) y Acatenango (3976 m), se localizan en la Cadena Volcánica. Aquí existen bosques mixtos caracterizados por Abies, Alnus, Buddleja, Juniperus y Pinus, distribuidos entre los 3000 y 4000 m de elevación.

La mayor parte de la zona de estudio ha sido influenciada por la acción del hombre. La intervención antropógena es muy evidente en la Sierra de los Cuchumatanes, donde se encuentran asentamientos humanos en los alrededores de las cumbres de las montañas. La actividad del hombre incluye la tala (para construcción y leña), la quema, la ganadería (principalmente de ganado ovino), y cultivos de papa y avena. En síntesis, la presencia humana ha cambiado drásticamente las condiciones de ecosistemas naturales. Es por esto que pocos de los ambientes de alta montaña en Guatemala se encuentran en un estado inalterado (Islebe, 1993).

\title{
METODOLOGIA
}

Entre 1991 y 1992 se efectuaron varios inventarios geobotánicos en las partes altas de la Sierra de los Cuchumatanes y de la Cadena Volcánica. Con base en las colecciones de muestras de plantas se elaboró una lista florística preliminar que fue actualizada y complementada a través de consultas bibliográficas.

En este listado se presentan en orden alfabético las familias, géneros y especies de las plantas leñosas, de manera similar a lo elaborado por Kappelle et al. (1991). Los Eupatorieae siguen la nomenclatura de King y Robinson (1987). Los binomios se acompañan de los nombres de sus autores abreviados de acuerdo con las recomendaciones de Halliday et al. (1980).

\section{RESULTADOS}

Se determinaron 112 especies leñosas, agrupadas en 39 familias y 63 géneros diferentes.

\author{
Intervalo de distribución \\ altitudinal $(\mathrm{m})$
}

\author{
SPERMATOPHYTA \\ GYMNOSPERMAE \\ CUPRESSACEAE (2) \\ Cupressus lusitanica Miller \\ Juniperus standleyi Steyermark
}

2000-3200

3000-4000 
PINACEAE (5)

Abies guatemalensis Rehder

2600-3400

Pinus ayacahuite Ehrenberg

2500-3300

$P$. hartwegii Lindl.

2800-3900

$P$. montezumae Lambert

2500-3200

P. pseudostrobus Lindl.

1000-3000

PODOCARPACEAE (1)

Podocarpus oleifolius D. Don

2000-3200

TAXACEAE (1)

Taxus globosa Schlecht.

2200-3000

\section{ANGIOSPERMAE}

MONOCOTYLODONEAE

AGAVACEAE (2)

Agave hurteri Trelease

1000-3500

Yucca elephantipes Regel

1500-3100

POACEAE (2)

Chusquea lanceolata Hitchc.

2000-3000

C. Iongifolia Swallen

2000-3000

\section{DICOTYLEDONEAE}

APIACEAE (1)

Myrrhidendron donnell-smithii J. Coulter \& Rose

2500-3200

AQUIFOLIACEAE (3)

Ilex anodonta Standl. \& Steyerm.

1300-3000

I. gracilipes I. M. Johnston

1700-3000

I. tolucana Hemsl.

1500-3000

ARALIACEAE (1)

Oreopanax xalapensis (Kunth) Dcne. \& Planchon

2500-3200

ASTERACEAE (15)

Ageratina mairetiana (DC.) R. King \& H. Robinson

3000-3300

A. pazcuarensis (B. Robinson) R. King \& H. Robinson

2800-3400

Archibaccharis serratifolia (Kunth) Blake

2400-3000

Baccharis conferta Kunth

1900-3000

$B$. heterophylla Kunth

2000-3000 
Islebe et al.: Especies Leñosas de la Sierra de los Cuchumatanes y de la Cadena Volcánica

B. salicifolia (Ruiz \& Pavon) Pers.

2000-3000

Bartlettina oresbioides (B. Robinson) R. King \& H. Robinson 2900-3300

Chromolaena collina (DC.) R. King \& H. Robinson 2800-3200

Oxylobus arbutifolius (Kunth) Gray

$3000-3400$

O. glanduliferus (Sch. Bip.) Gray

$3000-4000$

Roldana angulifolia (DC.) H. Robinson \& R. D. Brettell

$2500-3200$

$R$. barba-johannis (DC.) H. Robinson \& R. D. Brettell

$2600-3200$

Senecio mairetianus DC.

$2900-3200$

Stevia polycephala Bertol.

$2600-3500$

Verbesina calciphila Standl. \& Steyerm.

$3200-3500$

BERBERIDACEAE (1)

Mahonia volcanica Standl. \& Steyerm.

$3100-3600$

BETULACEAE (3)

Alnus firmifolia Fernald

$2600-3500$

A. arguta (Schlecht.) Spach

$1300-3000$

Ostrya virginiana var. guatemalensis (Winkl.) Macbride

$1000-3000$

BRUNELLIACEAE (1)

Brunellia mexicana Standl.

$2000-3000$

CAPRIFOLIACEAE (3)

Sambucus mexicana Presl

2000-3400

Symphoricarpos microphyllus Kunth

$2700-3400$

Viburnum euryphyllum Standl. \& Steyerm.

2500-3200

CLETHRACEAE (1)

Clethra pachecoana Standl. \& Steyerm.

$2500-3000$

CORIARIACEAE (1)

Coriaria ruscifolia ssp. microphylla

$2000-3000$

(Poir) L. Skog

CORNACEAE (1)

Cornus disciflora Mociño \& Sessé ex DC.

2500-3000

CUNONIACEAE (2)

Weinmannia pinnata Engler

2000-3000

W. tuerckheimii Engler

2000-3000

ERICACEAE (13)

Arbutus xalapensis Kunth

$2800-3500$

Comarostaphylis arbutoides Lindl.

$3000-3300$

C. cratericola Donn.-Sm.

$3000-3800$ 
C. pyrifolia Small

3000-3200

Pernettya prostrata (Cav.) DC.

2600-4100

$P$. saxicola Standl. \& Steyerm.

3500-4000

Vaccinium breedlovei L. Wms.

2500-3000

V. confertum Kunth

2600-3500

V. consanguineum Kunth

2000-3000

V. geminiflorum Kunth

3000-4100

V. leucanthum Cham. \& Schlecht.

2000-3000

V. matudae Lundell

1700-3300

V. minarum Standl. \& Steyerm.

2000-3200

EUPHORBIACEAE (2)

Acalypha trachyloba Muell. Arg.

1800-3100

A. triloba Muell. Arg.

2500-3000

FAGACEAE (3)

Quercus acatenangensis Trelease

1500-3200

Q. borucasana Trelease

2500-3200

Q. peduncularis Née

1000-3000

GARRYACEAE (2)

Garrya corvorum Standl. \& Steyerm.

3000-3500

G. laurifolia Hartweg ex Benth.

2200-3300

LAURACEAE (5)

Litsea glaucescens Kunth

1300-3100

L. guatemalensis Mez

1500-3000

L. neesiana (Schauer) Hemsl.

1900-3000

Ocotea chiapensis (Lundell) Standl. \& Steyerm.

1500-3000

Phoebe salvini (Mez) Lundell

1800-3200

LABIATAE (5)

Salvia areolata Epling

2600-3100

S. disjuncta Fernald

2500-3100

S. excelsa Benth.

2500-3100

S. lasiantha Benth

2500-3000

S. lavanduloides Kunth

2000-3000

LEGUMINOSAE (1)

Lupinus montanus Kunth

$3000-4000$

LOGANIACEAE (3)

Buddleja megalocephala Donn.-Sm.

2400-3600

$B$. nitida Benth.

2000-3200

B. skutchii Morton

1600-3200 
Islebe et al.: Especies Leñosas de la Sierra de los Cuchumatanes y de la Cadena Volcánica

MELASTOMATACEAE (1)

Miconia sp.

$1000-3000$

ONAGRACEAE (2)

Fuchsia microphylla Kunth

2500-3400

F. splendens Zucc.

2900-3200

PAPAVERACEAE (1)

Bocconia vulcanica Donn.-Sm.

$2500-3200$

POLYGALACEAE (1)

Monnina xalapensis Kunth

$1200-3000$

POLYGONACEAE (1)

Muehlenbeckia thamnifolia (Kunth) Meissner

$1800-3000$

PYROLACEAE (1)

Chimaphila umbellata (L.) Barton

$2000-3000$

RHAMNACEAE (4)

Ceanothus coeruleus Lagasca

2000-3000

Rhamnus capreaefolia Schlecht.

$1200-3000$

$R$. nelsoni Rose

$1900-3000$

$R$. serrata Willd. ex Roem. \& Schult.

$3300-3500$

ROSACEAE (7)

Acaena elongata L.

2000-3500

Holodiscus argenteus (L. f.) Maxim.

2900-4000

Prunus capuli Cav.

2000-3000

$P$. rhamnoides Koehne

2200-3000

Rubus eriocarpus Liebm.

2500-3100

$R$. hadrocarpus Standl. \& Steyerm.

2500-3000

$R$. trilobus Seringe

2800-3300

SAURAUIACEAE (3)

Saurauia cuchumatanensis Standl. \& Steyerm.

2000-3000

S. oreophila Hemsl.

$1500-3300$

S. subalpina Donn.Sm.

$1400-3300$

SAXIFRAGACEAE (2)

Ribes ciliatum Humb. \& Bonpl. ex Roem. \& Schult. 2800-3400

R. microphyllum Kunth

2800-3400

SCROPHULARIACEAE (1)

Penstemon gentianoides (Kunth) Poiret

$2800-3600$ 
SOLANACEAE (5)

Cestrum anagyris Dunal $\quad 2500-3200$

C. dasyanthum Kunth $2500-3200$

C. roseum Kunth $2500-3000$

Solanum cervantesii Lagasca 2000-3200

S. muencheri Standl. \& Steyerm. 3000-3700

SYMPLOCACEAE (3)

Symplocos abietorum Standl. \& Steyerm. $\quad 3000-3300$

S. hartwegii A. DC. $\quad 1200-3400$

S. vatteri Standl. \& Steyerm. 2800-3300

WINTERACEAE (1)

Drimys granadensis L. $\quad 1600-3000$

\section{DISCUSION Y CONCLUSIONES}

Del total (112) de las especies leñosas presentes en la zona de estudio, 9 son gimnospermas repartidas en cuatro familias (Cupressaceae, Pinaceae, Podocarpaceae y Taxaceae), 4 son monocotiledóneas (Agavaceae y Poaceae) y 99 son dicotiledóneas. Las familias mejor representadas son las Ericaceae (11\%) y las Asteraceae (16\%). Otros grupos de substancial abundancia son las Pinaceae (4\%) y Rosaceae (6\%). Los géneros con mayor número de especies son Vaccinium (7), Pinus (4) y Quercus (3).

Las condiciones ecológicas que determinan la distribución de los bosques de Abies guatemalensis se asemejan a aquellas que influyen en la repartición de los de Abies religiosa del centro de México. Más específicamente, los bosques de Abies, tanto en Guatemala como en México, son favorecidos por suelos bien drenados y húmedos y por la frecuencia y permanencia de nubosidad (Madrigal, 1967; Lauer, 1978; Velázquez y Cleef, 1993). Además, su distribución se restringe principalmente a las elevaciones entre los 3000 y $3500 \mathrm{~m}$. Similarmente, el ambiente relativamente seco de los lugares donde se establece el bosque mixto de Juniperus standleyi-Pinus hartwegii también se asemeja a las condiciones típicas de los bosques mixtos de Alnus firmifolia-Pinus spp. de Guatemala y del centro de México (Velázquez y Cleef, 1993; Islebe y Velázquez, en prensa). Se recomienda realizar estudios encaminados a cuantificar los límites mínimos tolerables de humedad de estas asociaciones.

No se han elaborado hasta ahora comparaciones detalladas entre tipos de vegetación (comunidades) afines de Guatemala y otros países centroamericanos (Islebe y Kappelle, 1994). A nivel genérico se estima que Guatemala y México comparten cerca de $95 \%$ de sus floras. Rzedowski (1991) propone áreas de referencia para delimitar regiones con base en sus características naturales, por ejemplo las especies vegetales endémicas. Según Rzedowski (1991), Guatemala forma parte de la zona fitogeográfica "Megaméxico 2", que incluye todo el territorio mexicano y se extiende hasta el norte de Nicaragua. El límite meridional de la distribución de las pináceas se encuentra en el norte de Nicaragua (Rzedowski, 1978). Los resultados de este estudio aportan más evidencia en favor de esta regionalización propuesta por Rzedowski (1991). 
Islebe et al.: Especies Leñosas de la Sierra de los Cuchumatanes y de la Cadena Volcánica

Veblen (1976) e Islebe (1993) enfatizaron la necesidad de implementar medidas de conservación urgentes para las zonas montañosas de Guatemala. Los autores esperan que la información aquí presentada contribuya al conocimiento de la diversidad biológica de Guatemala y que ésta sea utilizada para la elaboración de planes de manejo y conservación.

\section{AGRADECIMIENTOS}

Se agradece la colaboración recibida durante el trabajo de campo a las siguientes personas: Renate Hübner, Cruz Jerónimo Carrillo, Feliciano García, José Pérez y Frank Gernaert. El primer autor recibió apoyo financiero de la fundación "Gottlieb Daimler und Karl Benz Stiftung", Ladenburg, Alemania. Harold Robinson identificó algunas Asteraceae. Maarten Kappelle aportó comentarios valiosos al manuscrito. La cooperación del CONAP, Guatemala, fue esencial para el desarrollo del presente estudio.

\section{LITERATURA CITADA}

Bartram, E. B. 1949. Mosses of Guatemala. Chicago Natural History Museum, Fieldiana, Botany. Vol. 25. $442 \mathrm{pp}$.

Gentry, A. H. 1978. Floristic knowledge and needs in pacific tropical America. Brittonia 30(2): 134-153.

Halliday, P., R. D. Meikle, J. D. Story y H. Wilkinson. 1980. Draft index of author abbreviations. The Herbarium, Royal Botanic Gardens. Kew. 257 pp.

Islebe, G. A. 1993. Will Guatemala's Juniperus-Pinus forests survive? Environmental Conservation 20: 167-168.

Islebe, G. A. y M. Kappelle. 1994. A phytogeographical comparison between subalpine forests of Guatemala and Costa Rica. Feddes Repertorium Specierum Novarum 105: 73-87.

Islebe, G. A. y A. Velázquez. (En prensa). Affinity among mountain ranges in Megamexico: a phytogeographical scenario. Vegetatio.

Kappelle, M., N. Zamora y T. Flores. 1991. Flora leñosa de la zona alta (2000-3819 m) de la Cordillera de Talamanca, Costa Rica. Brenesia 34: 121-144.

King, R. M. y Robinson, H. 1987. The genera of the Eupatorieae (Asteraceae). Monogr. Syst. Bot. Missouri Bot. Gard. 22: 1-576.

Lauer, W. 1978. Timberline studies in central Mexico. Arctic and Alpine Research 10(2): 383-396.

Madrigal, J. 1967. Contribución al conocimiento de la ecología de los bosques de oyamel (Abies religiosa (H.B.K.) Schl. et Cham.) en el Valle de México. Instituto Nacional de Investigaciones Forestales. Boletín Técnico No. 18. México, D.F. 94 pp.

Rodríguez, M. L. 1921. Note sur le végétation du Guatemala. Bull. Soc. Bot. France 67: 356-360.

Rzedowski, J. 1978. Vegetación de México. Editorial Limusa. México, D.F. 432 pp.

Rzedowski, J. 1991. Diversidad y orígenes de la flora fanerogámica de México. Acta Bot. Mex. 14: 3-21.

Sapper, K. 1894. Grundzüge der physischen Geographie von Guatemala. Petermanns Mitteil., Ergänzungsheft No. 113. 30 pp.

Sapper, K. 1897. Das nördliche Mittelamerika. Braunschweig. 118 pp.

Sapper, K. 1902. Mittelamerikanische Reisen und Studien. Braunschweig. 140 pp.

Smith, J. D. 1889-1907. Enumeratio plantarum guatemalensium. Oquawka, III. 8 vols.

Standley, P. C. y J. A. Steyermark. 1945. The vegetation of Guatemala, a brief review. In: Plants and plant sciences in Latin America. Chronica Botanica Co. Waltham, Mass. pp. 275-278.

Standley, P. C. y L. O. Williams. 1946-77. Flora of Guatemala. 13 parts. Illus. (Fieldiana; Bot., 24). Chicago. 
Veblen, T. T. 1976. The urgent need for forest conservation in highland Guatemala. Biol. Conserv. 9: $141-154$

Velázquez, A. y A. M. Cleef. 1993. Plant communities of Tláloc and Pelado volcanoes, Mexico. Phytocoenologia 22: 145-191.

Wagner, M. 1866. Über den Character und Höhenverhältnisse der Vegetation in den Cordilleren von Veragua und Guatemala. Sitz. Ber. Kgl. Bayr. Akad. Wiss. 1: 1-13. 